Published in Hot Cracking Phenomena in Welds II, 2008, Springer, Eds :T. Bollinghaus, H. Herold, C. Cross and J. Lippold, pp. 19-37

\title{
APPLICATION OF THE RAPPAZ DREZET GREMAUD HOT TEARING CRITERION TO WELDING OF ALUMINIUM ALLOYS
}

\author{
J.-M. DREZET* and D. ALLEHAUX** \\ *Ecole Polytechnique Fédérale de Lausanne, Laboratoire de Simulation \\ des Matériaux, station 12, CH-1015 Lausanne, Tel. +41 2169339 20, \\ jean-marie.drezet@epfl.ch
}

**EADS Corporate Research Centre, Paris, F-92152 Suresnes, France Tel. +331469730 92, delphine.allehaux@eads.net

\begin{abstract}
Hot tearing, a severe defect occurring during solidification is the conjunction of tensile stresses which are transmitted to the mushy zone by the coherent solid underneath and of insufficient liquid feeding to compensate for the volumetric change. The RDG (Rappaz Drezet Gremaud) criterion for the appearance of hot tears in metallic alloys [1] is based upon a mass balance performed over the liquid and solid phases and accounts for the tensile deformation of the solid skeleton perpendicular to the growing dendrites and for the induced interdendritic liquid feeding. When tackling the problem of hot tearing in welding of aluminium alloys, the RDG criterion can be used at three levels of increasing complexity by:

- ranking the alloy with regards to their sensitivity to hot cracking

- studying the risk of hot tearing in the process using only the thermal field (thermal criterion),

- and studying the influence of the mechanical behaviour of the mushy alloy on the risk of hot cracking (thermo-mechanical criterion).

Each level is illustrated by an example dealing with laser beam welding. Nevertheless, one of the critical issues in the RDG approach is the definition of a coherency point which, in low-concentration alloys, corresponds
\end{abstract}


to the bridging or coalescence of the primary phase. To tackle this aspect, a 2D granular model is presented together with preliminary results.

\section{Introduction}

Hot tearing together with microporosity have always been recognized as major defects in castings of aluminium alloys, the first one being typical of semi-continuous casting whereas the second one is dominant in shape castings. These two defects are also present in welds. As mentioned by Campbell [2], they are interconnected, as both result from a lack of feeding and nucleation of a pore/void in the remaining liquid. However, if porosity is associated with solidification shrinkage and can occur within the grains or at grain boundaries, hot tearing is clearly linked with tensile stresses in the solid and is confined to grain boundaries.

An excellent review of all the hot tearing models has been published by Eskin et al. [3] in 2004. Recent developments made both at the macroscopic and microscopic levels, have pointed out the difficulties in connecting these two scales. Macroscopic approaches are based nowadays on twophase approaches [4-6]: they are the natural extension of the so-called RDG criterion [1], but using a more rigorous rheological approach for the compressible mushy solid phase. The main advantage of such methods is that they can take into account the whole scale of the parts to be welded, but their weakness is that averages can hardly account for the localization of strains and feeding at grain boundaries. On the microscopic scale, coalescence and percolation of grains can be accounted for at the scale of a small volume element of the mushy zone (typically a few cubic centimetres) using granular approaches [7-9]. Although such techniques cannot yet consider a whole solidification process, they provide a detailed and interesting view of the phenomena occurring during hot tearing, in particular localization of strains and feeding, gradual transition from a continuous liquid film network to a fully coherent solid, etc. These methods are briefly presented in the last section.

\section{The RDG hot tearing criterion}

In the RDG criterion [1], a deformation perpendicular to the thermal gradient is applied to the solid phase, regardless whether it corresponds to columnar or equiaxed dendrites (see Fig. 1). The component of the strains 
parallel to the thermal gradient is neglected on the basis that it is not a component susceptible of inducing hot tearing. Defining then the average density of the solid-liquid mixture as $\rho=\rho_{s} g_{s}+\rho_{\ell} g_{\ell}$, where $g_{v}$ and $\rho_{v}$ are the volume fraction and the density of phase $v(v=s, \ell)$, and using Darcy's equation describing the interdendritic flow in a mushy zone, the following expression is derived for unsteady conditions $[1,10]$ :

$$
\rho \frac{\partial g_{s}}{\partial t}+(1+\beta) g_{s}\left(\dot{\varepsilon}_{y y}+\dot{\varepsilon}_{z z}\right)-\operatorname{div}\left(\frac{K}{\mu}\left(\operatorname{gradP} P_{l}-\rho_{l} g\right)\right)=0 .
$$

$\beta=\left(\rho_{\mathrm{s}} / \rho_{\ell}-1\right)$ is the solidification shrinkage, $\dot{\varepsilon}_{y y}$ and $\dot{\varepsilon}_{z z}$ are the two components of the strain rate of the solid perpendicular to the thermal gradient (see Fig. 1), $\mathrm{K}$ is the permeability of the mush, $\mu$ is the viscosity and $\mathrm{p}_{\ell}$ the local pressure in the interdendritic liquid, $\mathbf{g}$ being the gravity vector. Note that $\rho_{\mathrm{s}}$ and $\rho_{\ell}$ have been assumed constant, while $g_{\mathrm{s}}$ is constant in the directions perpendicular to the thermal gradient (directional solidification). It is interesting to notice that although a deformation is applied, it is the strain rate that appears in Eq. 1. This expression is fairly general and can be interpreted as follows: solidification shrinkage $\left(1^{\text {st }}\right.$ term $)$ and/or deformation of the solid ( $2^{\text {nd }}$ term) have to be compensated by liquid flow ( $3^{\text {rd }}$ term) if pores or hot tears are to be avoided. In the case a third phase (pores or hot tears) is considered, the right hand term of Eq. 1 is simply replaced by - $\partial \mathrm{g}_{\mathrm{p}} / \partial \mathrm{t}$, where $\mathrm{g}_{\mathrm{p}}$ is the fraction of pores (or hot tears) [11].

Under steady directional solidification conditions at a velocity $\mathrm{v}_{\mathrm{T}}$, the maximum pressure drop, $\Delta \mathrm{p}_{\max }$, across the mushy zone and associated with deformation and solidification shrinkage is given by:

$$
\Delta p_{\max }=(1+\beta) \mu \int_{0}^{L} \frac{E}{K} d x+v_{T} \beta \mu \int_{0}^{L} \frac{g_{l}}{K} d x
$$

where $\mathrm{E}$ is the cumulated strain rate $E(x)=\int g_{s}\left(\dot{\varepsilon}_{y y}+\dot{\varepsilon}_{z z}\right) d x$. These two integrals can be transformed into integrals over temperature, thus introducing a "competition" between strain rate and thermal gradient, G, for the first contribution, and the standard $\mathrm{v}_{\mathrm{T}} / \mathrm{G}$ ratio for the shrinkage term as already derived by Niyama in his porosity criterion [12]. 


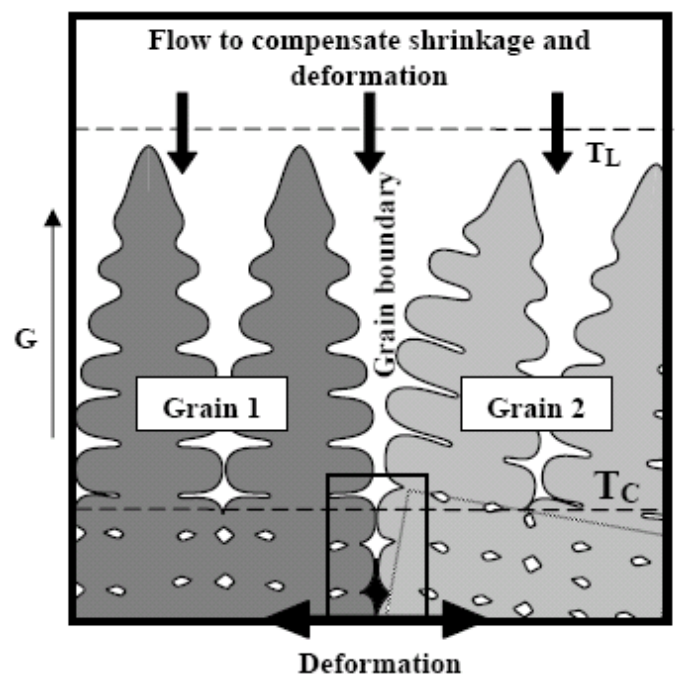

Figure 1: Schematics of hot tearing formation and of the two-phase problem considered in the RDG approach [11].

The RDG criterion simply states that a hot tear forms if the local pressure in the liquid, i.e., the metallostatic pressure minus the pressure drop, falls below a given cavitation pressure. This criterion has nevertheless a few limitations:

- using only the perpendicular component of the plastic strains is not strictly valid, the longitudinal component also inducing some suction (or expulsion) of the liquid;

- the lower bound of the integrals of Eq. (2) is ill-defined. As gs tends towards unity, the permeability goes to zero and the calculation diverges. In practical situations, this bound is set up to a value of $g_{s}$ at which the solid is considered as coherent, i.e., the liquid remains only as liquid pockets (no continuous liquid films).

- in relation to grain boundaries, the method does not consider any localization of the strains and feeding.

Stating the local pressure in the liquid films must not fall below a cavitation pressure if no hot tears should form is equivalent to state that the strain rate must remain lower than a maximum value. In other words, the mush can sustain some deformation but its rate should remain low enough in order to permit liquid feeding. The deformation is indeed limited by the ductility of the solid + liquid mixture. Ductility curves obtained by tensile tests exhibit the typical shape of $U$ when liquid and solid phases are present as shown in fig. 2 [13-14]. Some ductility is present at temperatures around the coherency temperature (beginning of mechanical resistance in 
tension), then decreases when approaching the solidus temperature before increasing again in the solid state. The brittle temperature range, BTR, corresponds to the temperature interval where the ductility is really low [15]. In order to avoid hot cracking, the thermomechanical path $\varepsilon(T)$ of the alloy must not cross the $\mathrm{U}$ ductility curve. In other words, the slope of $\varepsilon(T)$ is limited. Noticing that $\frac{\partial \varepsilon}{\partial T}=\frac{\partial \varepsilon}{\partial t} * \frac{\partial t}{\partial T}=\frac{\dot{\varepsilon}}{\dot{T}}$, the strain rate is limited too, as found in the RDG approach. Note that the quantity $\frac{\dot{\varepsilon}}{\dot{T}}$ has also been used as a hot tearing indicator [3].

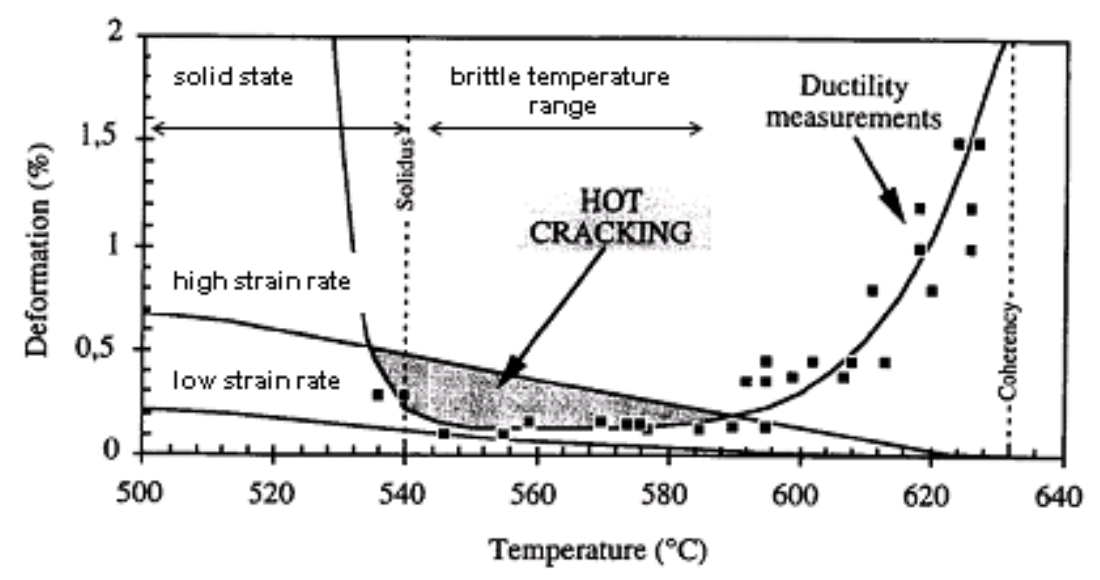

Figure 2: U ductility curve for the Al-Cu 4.5 wt. pct. alloy together with two thermomechanical paths (adapted from [14]).

Finally, the RDG criterion, originally derived for aluminium alloys, has been extended to steel by Drezet et al. [16] by taking into account the peritectic reaction that transforms ferrite into austenite. Moreover, Rindler et al. [17] have further worked out the criterion for steels by releasing the assumption of a uniform strain rate acting over the mush.

\section{Alloy hot tearing susceptibility}

Under the assumption that the strain rate applied to the mushy zone is uniform $\dot{\varepsilon}=\dot{\varepsilon}_{y y}+\dot{\varepsilon}_{z z}$ and that steady state is reached, the liquid pressure 
drop through the mush is made out of two contributions, $\Delta \mathrm{p}_{\mathrm{sh}}$ due to the solidification shrinkage and $\Delta \mathrm{p}_{\text {mec }}$ due to deformation [10]. The two contributions can be written as:

$$
\begin{array}{r}
\Delta \mathrm{p}_{\mathrm{sh}}+\Delta \mathrm{p}_{\mathrm{mec}}=\frac{180 \mu}{\mathrm{G} \lambda_{2}^{2}}\left[\mathrm{v}_{\mathrm{T}} \beta \mathrm{A}+\frac{(1+\beta) \mathrm{B} \dot{\varepsilon}}{\mathrm{G}}\right] \\
\text { with } \mathrm{A}=\int_{\mathrm{T}_{\mathrm{cg}}}^{\mathrm{T}_{\text {liq }}} \frac{\mathrm{f}_{\mathrm{s}}^{2} \mathrm{dT}}{\left(1-\mathrm{f}_{\mathrm{s}}\right)^{2}} \quad \text { and } \mathrm{B}=\int_{\mathrm{T}_{\mathrm{cg}}}^{\mathrm{T}_{\text {liq }}} \frac{\mathrm{f}_{\mathrm{s}}^{2} \int_{\mathrm{cg}}^{\mathrm{T}} \mathrm{f}_{\mathrm{s}} \mathrm{dT}}{\left(1-\mathrm{f}_{\mathrm{s}}\right)^{3}} \mathrm{dT}
\end{array}
$$

$\lambda_{2}$, the secondary dendrite arm spacing, is the typical length of the microstructure used to define the permeability $\mathrm{K}$. The two integrals A and B are related to microporosity formation induced by the solidification shrinkage and to hot tearing, respectively. The larger these quantities, the larger the pressure drop and therefore the higher the risk to initiate a hot tear. Note that the lower bound of the two integrals A and B are set to Tcg, the temperature at which coalescence of the grains occurs.

Within the framework of the European Wel-Air project [18], the RDG hot tearing criterion is used to analyse the influence of the alloy composition per se and of the filler material on the hot tearing susceptibility for new generation aircraft aluminium alloys. To do so, the solidification path of the alloy (plus the filler material) was obtained using ProPHASE, a microsegregation program developed at Alcan Péchiney [19]. As the solidification path should be relevant with the solidification conditions undergone by the alloy during laser welding, a Scheil approach (no solid-state diffusion) is used owing to the very high cooling rate experienced by the alloy. The grain coalescence temperature is not easy to determine; the temperature corresponding to a solid fraction of $98 \%$ or the eutectic temperature if more than $2 \%$ eutectic has formed, was adopted as it correctly predicted the hot cracking susceptibility for binary alloys, the so-called $\Lambda$ curve [1]. Fig. 3 shows the solidification paths computed with ProPHASE for five aluminium alloys. Their composition is detailed in table 1. 


\begin{tabular}{|c|c|c|c|c|c|c|c|c|c|c|c|}
\hline Alloy & $\mathrm{Si}$ & $\mathrm{Fe}$ & $\mathrm{Cu}$ & $\mathrm{Mn}$ & $\mathrm{Mg}$ & $\mathrm{Cr}$ & $\mathrm{Zn}$ & $\mathrm{Ti}$ & $\mathrm{Zr}$ & $\mathrm{Sc}$ & $\mathrm{Li}$ \\
\hline AA2139 & 0.04 & 0 & 4.9 & 0.3 & 0.45 & 0 & 0 & 0 & 0 & 0 & 0 \\
\hline AA6056 & 0.85 & 0 & 0.95 & 0.45 & 0.73 & 0 & 0 & 0 & 0 & 0 & 0 \\
\hline AA6013 & 0.8 & 0 & 0.85 & 0.5 & 1 & 0 & 0 & 0 & 0 & 0 & 0 \\
\hline AA2098 & 0 & 0 & 3.66 & 0.01 & 0.33 & 0 & 0 & 0 & 0 & 0 & 0.99 \\
\hline AA2022 & 0 & 0 & 5.2 & 0.4 & 0.25 & 0 & 0 & 0 & 0 & 0 & 0 \\
\hline
\end{tabular}

Table 1: composition for five selected aluminium alloys.

As the end of solidification is particularly important, a zoom of the solidification paths is presented in fig. 4. The ranking of the alloys with respect to their hot cracking susceptibility HCS is presented in Fig. 5 where the integral A is represented, knowing that B varies in a very similar way. It appears that the alloys AA2139 and AA2098 exhibit the lowest hot tearing susceptibility. Albeit, this trend must be balanced by the fact that these alloys present other shortcomings such as equiaxed grain zone (EQZ) and possible liquation cracks.

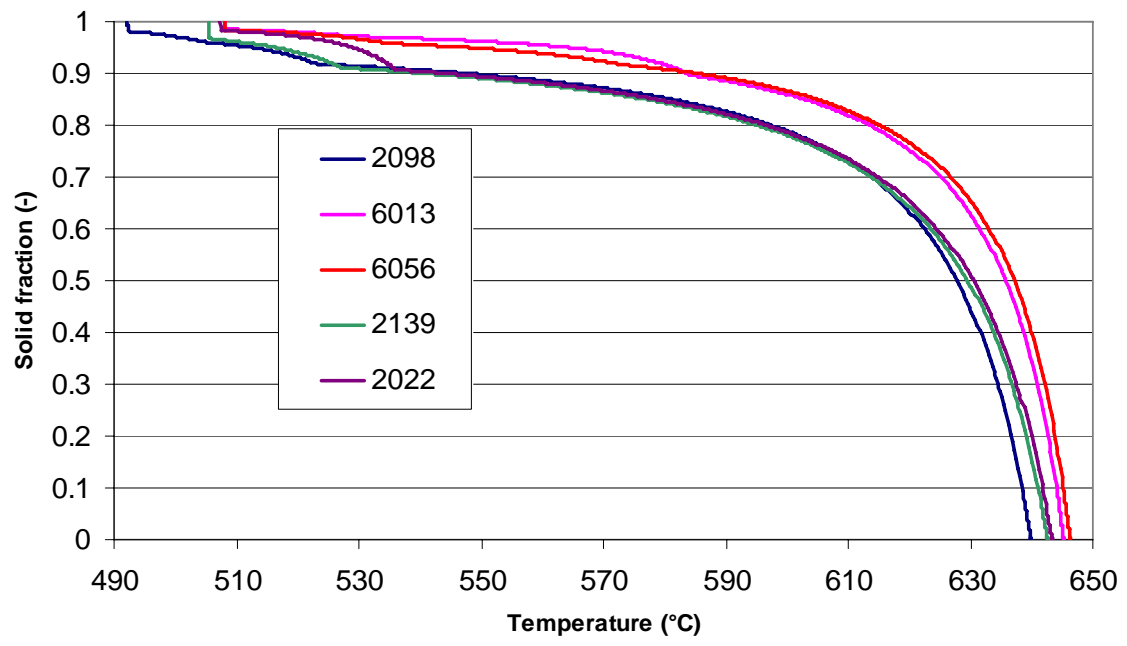

Fig. 3: Solidification paths of the Wel-Air alloys as computed by ProPhase. 


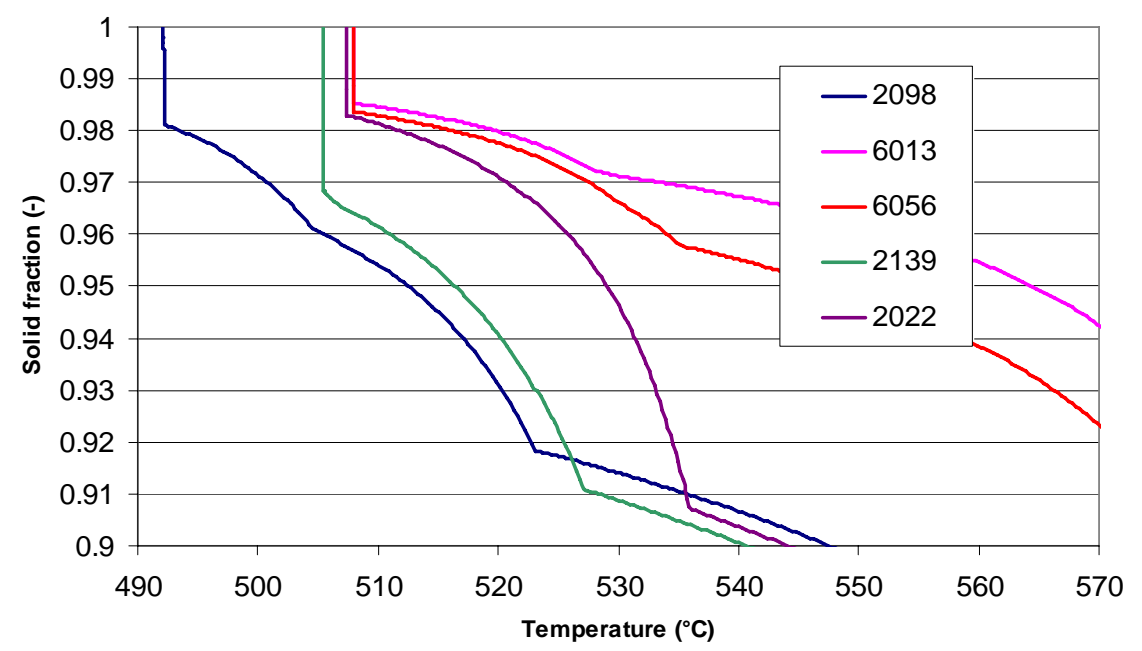

Fig. 4: Zoom at solid fractions higher than $90 \%$ of the solidification paths.

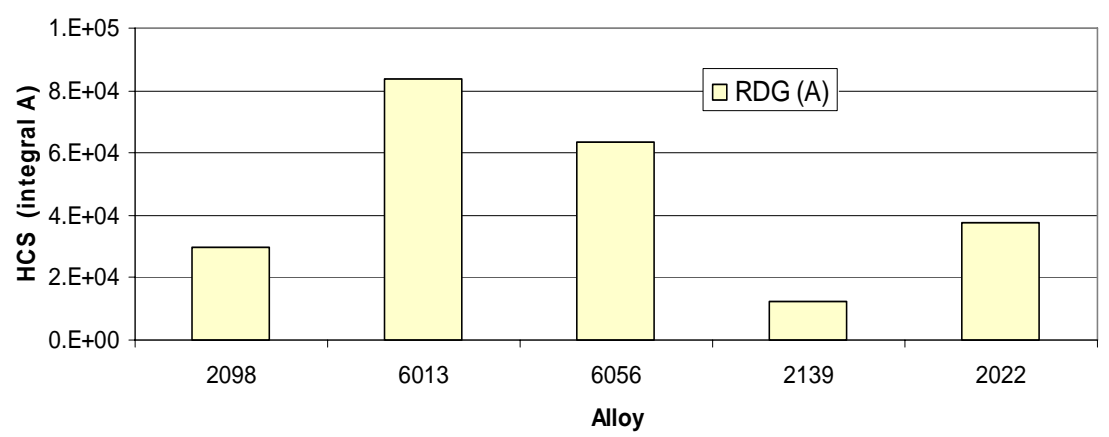

Fig. 5: HCS of the WEL-AIR alloys computed with the RDG criterion.

To improve the weldability of the aluminium alloys, it is common to bring a filler wire in different proportion to the weld bath, the idea being to increase the Si content in order to get more eutectic. Albeit, the welded material has lower mechanical properties and the use of filler material presents some technological constraints. The influence of the amount of filler material on hot tearing susceptibility is computed for the alloy AA2098. Fig. 6 shows the computed solidification paths of the 2098 alloy together with $15 \%, 25 \%$ and $33 \%$ of 4047 filler material and Fig. 7, the calculated hot cracking susceptibilities. It is interesting to notice that adding some silicon completely changes the nature of the phases; in particular the $\mathrm{Al}_{2} \mathrm{Li}_{3} \mathrm{Si}_{2}$ intermetallic phase appears when $\mathrm{Si}$ is present and the solidifica- 
tion ends at $501{ }^{\circ} \mathrm{C}$ with the deposition of $\mathrm{Al}_{2} \mathrm{Cu}$. This has a large influence on hot cracking as it decreases the HCS by almost a factor 6 . Nevertheless, this one remains almost unchanged when increasing the amount of 4047.

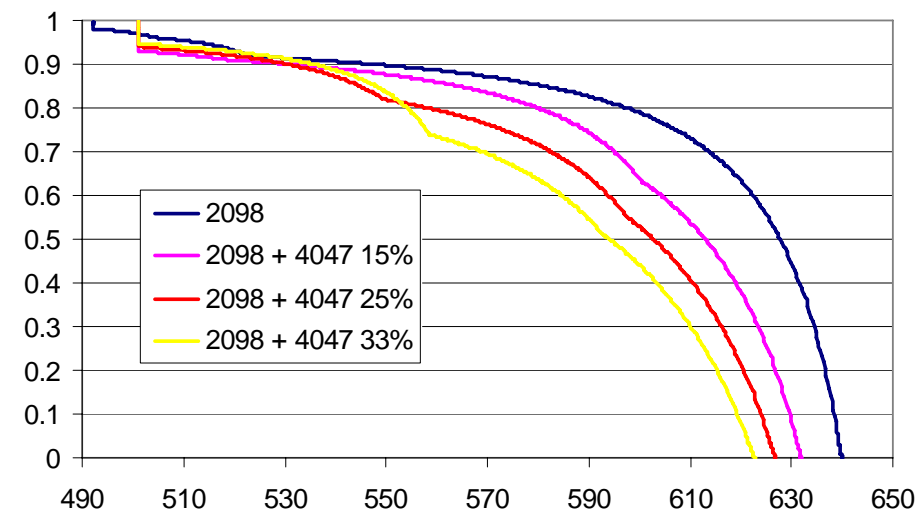

Fig. 6: solidification paths of AA2098 alloy plus AA4047 filler.

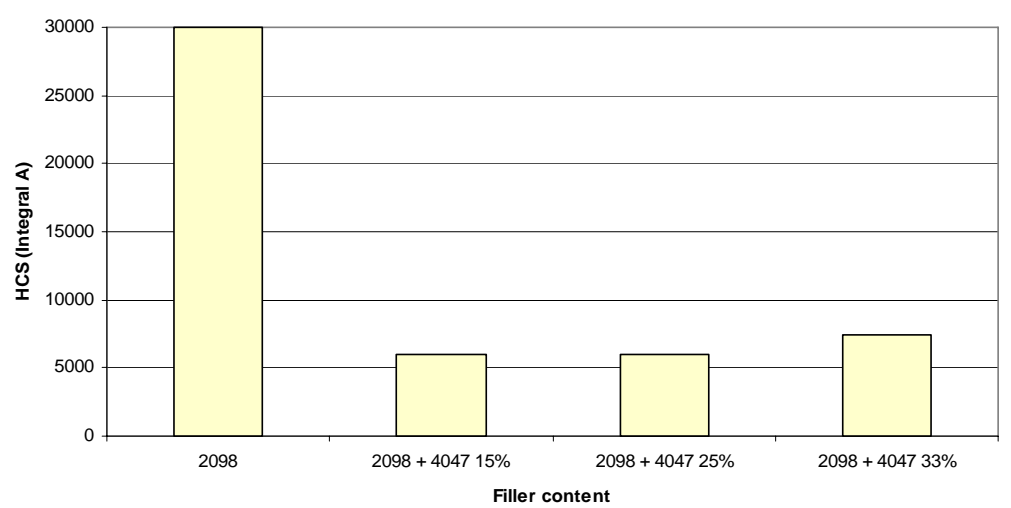

Fig. 7: Influence of the filler content on HCS.

\section{Thermal hot tearing criterion}

The thermal criterion allows us to compute the hot tearing susceptibility of a given alloy solidified under the conditions imposed by the process, i.e. in a given thermal gradient and with a given solidification speed. The hot cracking susceptibility, HCS, is therefore defined as the inverse of the maximum strain rate sustainable by the mushy alloy. Under 
steady state, this quantity is a function of the two integrals A and B but also of the local thermal gradient $\mathrm{G}$ and solidification speed $\mathrm{V}_{\mathrm{T}}$ :

$$
\begin{aligned}
& \Delta \mathrm{p}_{\max }=\Delta \mathrm{p}_{\mathrm{sh}}+\Delta \mathrm{p}_{\mathrm{mec}}-\rho \mathrm{gh} \leq \Delta \mathrm{p}_{\mathrm{cav}} \\
& \dot{\varepsilon}_{\mathrm{p}} \leq \dot{\varepsilon}_{\mathrm{p}}^{\max }\left(\mathrm{G}, \mathrm{V}_{\mathrm{T}}, \mathrm{A}, \mathrm{B}, \Delta \mathrm{p}_{\mathrm{cav}}, \lambda_{2}, \ldots\right) \\
& \mathrm{HCS}=\frac{1}{\dot{\varepsilon}_{\mathrm{p}}^{\max }}
\end{aligned}
$$

The computation of HCS for the AA6056 alloy (cf. table 1) welded at a speed of $1 \mathrm{~m} / \mathrm{min}$ with a $3 \mathrm{~kW}$ laser is presented as an example. The steady state temperature field in the butt joint is represented in Fig. 8. An eulerian approach is used, i.e. the material is transported at the laser speed under the fixed laser beam. The characteristics of the heat source can be found in [20]. Using the local solidification conditions along the coalescence isotherm, HCS is computed with the help of CalcoSOFT3D [21]. The results are shown in Fig. 9. As steady state is reached, HCS can be represented in a plane perpendicular to the laser speed. A maximum appears close to the bottom of the weld pool. Influence of the laser speed, power and heat source parameters can be assessed with such an approach.

Finally, note that the present approach does not require any mechanical computation, as the maximum strain rate the mush can sustain simply depends on thermal quantities (cf. Eq. 5). However, only steady state can be treated with the thermal criterion. The inclusion of a constitutive model for the mushy alloy and the investigation of transients are presented in the next section.

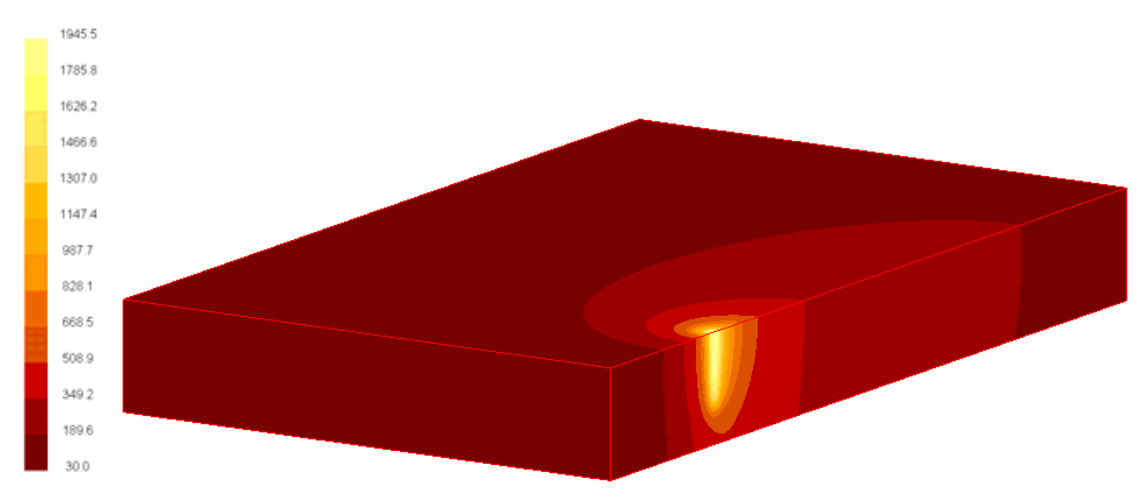

Fig. 8: steady state temperature field during laser welding of the AA6056 alloy. 

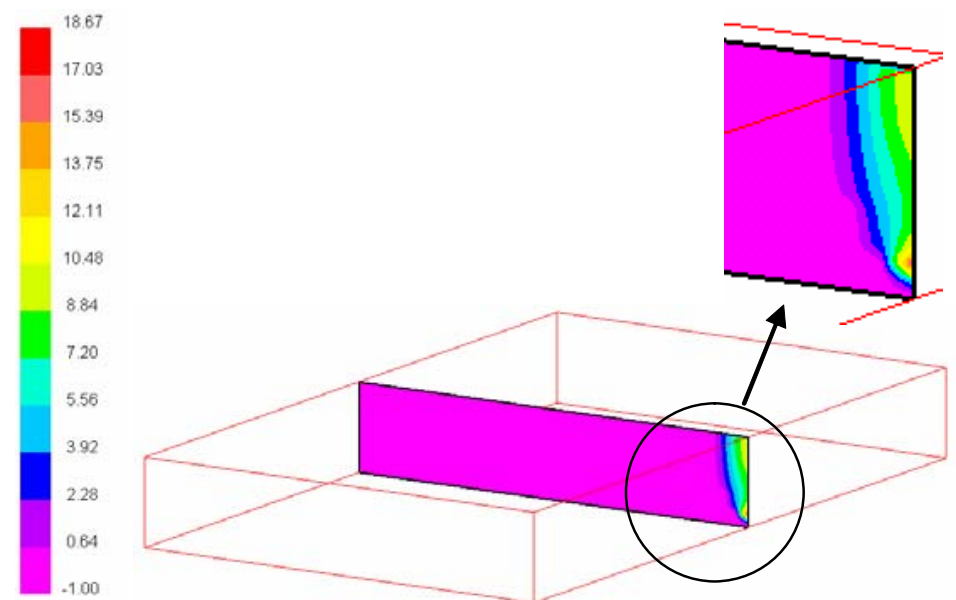

Fig. 9: Distribution of HCS.

\section{Thermo-mechanical approach}

By including the mechanical behaviour of the mush, the strain rate tensor undergone by the dendrites during solidification can be computed and therefore the induced pressure drop; the larger this value, the higher the risk to initiate a hot tear [22]. The main challenge is to establish a reliable constitutive model for the mechanical behaviour of the solidifying material. Although the mush should be treated as a compressible medium with the help of internal variable models [23], a simple incompressible model is adopted as a first step and for sake of simplicity. Indeed, many mechanical tests in the solidification interval are required to determine the numerous parameters describing the compressibility of the mushy alloy [23].

In this section, the mechanical strain rates undergone by the mushy zone at the rear of the weld pool are assessed in a simple configuration, the butt joint, in order to predict how process parameters can decrease the risk of hot tearing in steady state as well as transient regimes such as run-in and run-out. The results presented here deal again with the AA6056 alloy. Its BTR is considered to be $510^{\circ} \mathrm{C}-550^{\circ} \mathrm{C}$ and corresponds to solid fractions higher than $95 \%$ (cf. Fig. 4).

Thermal contraction arises as soon as the dendritic network is well developed and interconnected, that is at a solid fraction of $85 \%$ i.e. at a temperature of $600^{\circ} \mathrm{C}$ (coherency temperature). The rheology of the alloy is given by the classic viscoplastic Ludwik's model and is detailed in [20]. 
The mesh used for the butt joint configuration is presented in figure 10. Due to symmetry, only one half of the whole domain is meshed. The laser is supposed to travel along the z-axis and mesh is refined in the regions that undergo melting and solidification. The dimensions of the domain are given in fig. 10. The length of the domain (dimension in $\mathrm{z}$ ) was determined so that the transient run-in and run-out regimes are well separated by a steady state regime in the so-called on-going zone.

The laser heat input is modelled by a volume heat generation within a cylinder that is moving over the surface of the parts to be welded [20]. As the mechanical field does not influence the thermal field (owing to the absence of any air gap formation), the thermomechanical computation is uncoupled. The thermal field is computed first and then used as a loading for the mechanical calculation. As the two parts are tack welded prior to laser beam welding, they are considered to be part of the same continuum and therefore no contact elements are used. All plastic deformations are reset to zero above $600^{\circ} \mathrm{C}$, using the *anneal temperature feature in Abaqus 6.5 [24].

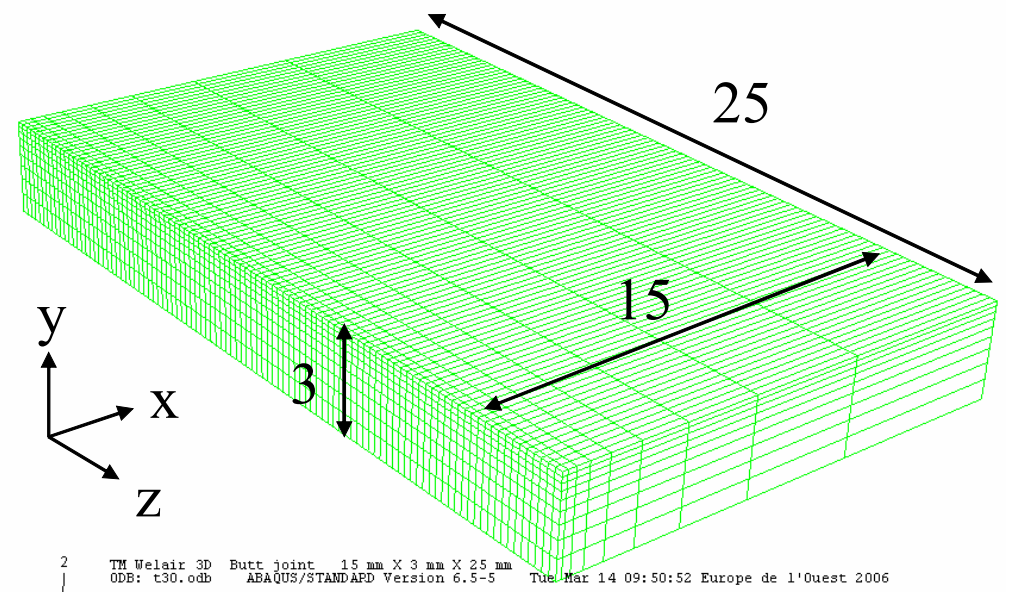

Fig. 10: FE mesh for the butt joint configuration (dimensions in $\mathrm{mm}$ ).

Following the Rappaz-Drezet-Gremaud [1] hot tearing criterion and the approach of Monroe and Beckerman [25], the larger the strain rate is, the higher the risk to initiate a crack. The mean and maximum values of the trace of the total (elastic + thermal + plastic) strain rate over the BTR are taken as hot tearing (HT) indicators: 


$$
\begin{aligned}
& \mathrm{HTI} 1=\underbrace{\text { Mean }}_{510^{\circ} \mathrm{C} \leq \mathrm{T} \leq 550^{\circ} \mathrm{C}}(\operatorname{tr} \dot{\varepsilon}) \\
& \text { HTI2 }=\underbrace{\operatorname{Max}}_{510^{\circ} \mathrm{C} \leq \mathrm{T} \leq 550^{\circ} \mathrm{C}}(\operatorname{tr} \dot{\varepsilon})
\end{aligned}
$$

These values are saved during cooling only (i.e. during solidification) and at each integration point using the user-subroutine UVARM of Abaqus 6.5. The larger this value, the higher the risk to initiate a hot crack.

The case presented here corresponds to a laser speed of $50 \mathrm{~mm} / \mathrm{s}$, i.e. 3 $\mathrm{m} / \mathrm{min}$, with a constant power all over the specimen length. The thermal field when the laser reaches the location $\mathrm{z}=18 \mathrm{~mm}$ is presented in Fig. 11. The weld pool corresponding to temperatures higher than $650^{\circ} \mathrm{C}$ is represented in grey.

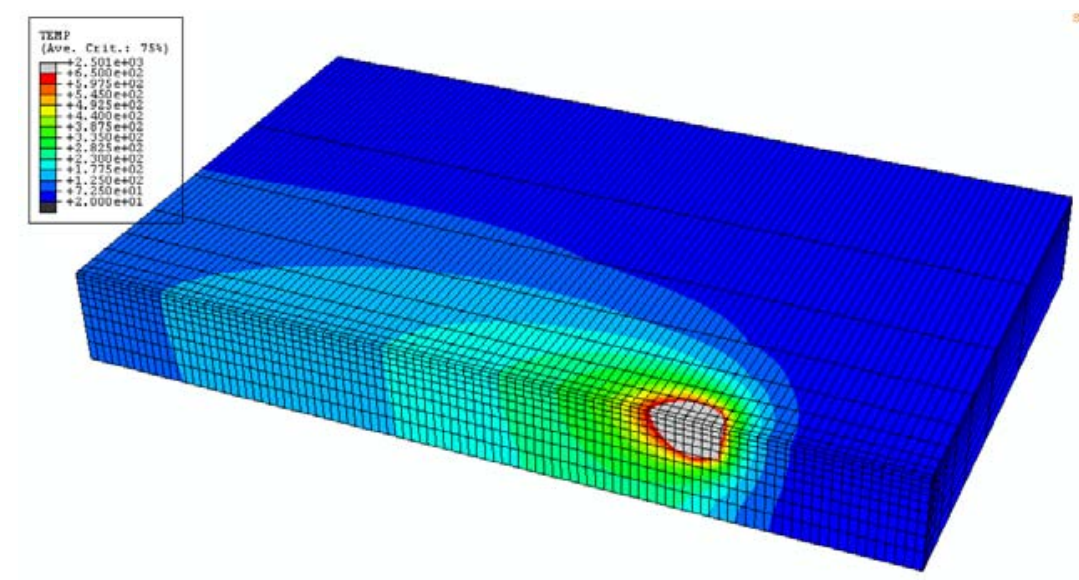

Fig. 11: Temperature distribution when the laser is located at $\mathrm{z}=18 \mathrm{~mm}$. The liquid pool appears in grey.

The distribution of the Von Mises equivalent plastic strain rate (positive quantity) and the two hot tearing indexes, HTI1 and HTI2, is presented in figure 12 as a function of the position, $\mathrm{z}$, along the specimen slightly below the top surface $(y=2.5 \mathrm{~mm}, \mathrm{x}=0)$. As expected, the three regimes of welding are evidenced: in the run-in, the strain rate and the hot tearing indexes exhibit high values; then they decrease and plateau in the on-going zone. Finally, in the transient run-out regime, the three quantities increase again. Both HTI1 and HTI2 present the same trends. One can notice that the equivalent plastic strain rate cannot be considered as an indicator for hot tearing since it is always positive by definition, whereas HTI1 and 
HTI2 can have negative values (i.e. no risk of hot cracking). This is the case when the strain rate reaches its maximum in the run-out whereas the two HT indexes get negative. In the steady state regime, HTI1 is $0.05 / \mathrm{s}$ whereas HTI2 is close to 0.16 /s. HTI1 and HTI2 both exhibit a maximum in the run-in and run-out regimes, which means that hot cracking is prone to occur at those locations. This is in accordance with industrial observations, where no hot tears are observed in the steady state regime but appear in the run-in and run-out. Finally, the HT indexes are larger in the run-in than the run-out, which seems to be less prone to hot cracking.

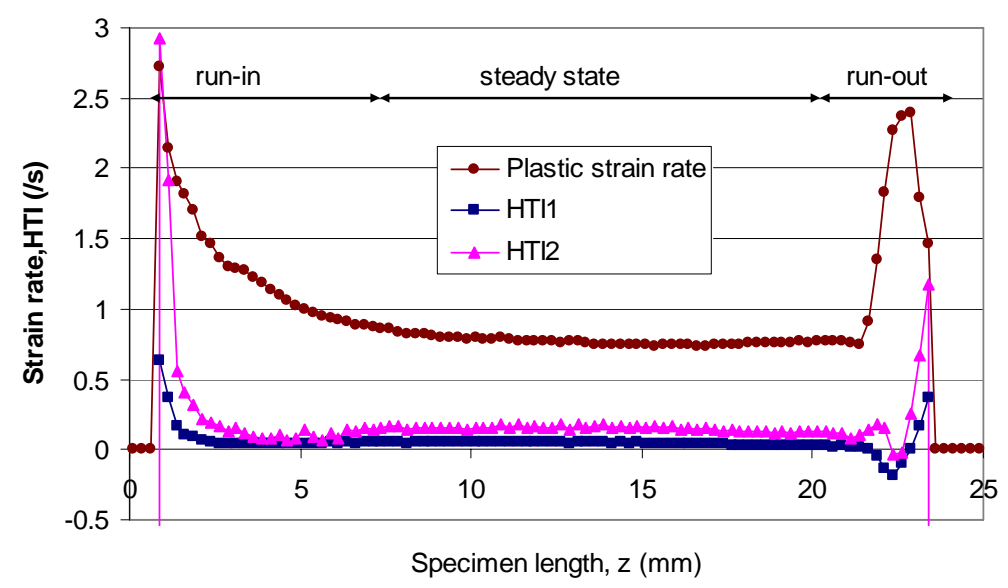

Fig. 12: Distribution of the equivalent plastic strain rate and HT indexes along the specimen.

\section{Granular model for hot tearing criterion}

As mentioned previously, one difficulty in the RDG approach is to estimate the temperatue at coalescence, Tcg that appears in Eq. 4. Indeed, average methods are unable to account for the localization of hot tears at grain boundaries. This localization is essentially due to the fact that liquid films remain to lower temperature as compared to those located in between dendrites of the same grains (Fig. 1). In other words, the formation of a coherent solid network by coalescence or bridging of dendrites arms occurs earlier within the grains as compared with grain boundaries. Rappaz et al. [26] have introduced for that purpose a coalescence or bridging undecooling which, for a pure metal, is given by: 


$$
\Delta T_{b}=\frac{1}{\Delta s_{f}} \frac{\gamma_{g b}-2 \gamma_{s l}}{\delta}
$$

where $\gamma_{\mathrm{gb}}$ is the grain boundary energy, $\gamma_{\mathrm{s} \ell}$ is the solid-liquid interfacial energy, $\Delta \mathrm{s}_{\mathrm{f}}$ is the volumetric entropy of fusion and $\delta$ is the thickness of the diffuse interfaces. For an alloy, coalescence is reached when a coalescence line (or surface) parallel to the liquidus, but $\Delta \mathrm{T}_{\mathrm{b}}$ below, is reached. Within a grain, there is no grain boundary energy and interfaces become attractive as soon as they get within interaction distance, i.e., distance $\delta$. At "repulsive" grain boundaries, $\gamma_{\mathrm{gb}}>2 \gamma_{\mathrm{se}}$, bridging is reached at some $\Delta \mathrm{T}_{\mathrm{b}}>0$.

This concept of bridging undercooling has been tested by experiment [27] where two crystals of a nickel-base superalloy are laser welded together under well defined conditions with increasing misorientations. At small misorientation (typically less than 15 deg.), no hot crack forms along the weld centreline, whereas at larger values, a crack is initiated under the same conditions, thus showing the influence of the grain boundary energy. The simulations done by molecular dynamics or by phase field are interesting but correspond to very small regions near the incoming interfaces, typically a few tens of nanometers or micrometers, respectively. In practical situations, hot tears are indeed located at grain boundaries, but the configuration of these boundaries associated with nucleation and growth of grains is essential. For this reason, a simplified approach of coalescence for a large population of equiaxed grains was undertaken first by Mathier et al. [7] and then by Vernède et al. [8-9]. In this granular approach, a random set of nucleation centres with random orientations is first generated in a given volume. Considering that the grains nucleate at the same time and that the temperature difference across each grain is small with respect to the growth undercooling, the grain boundaries correspond to the Voronoi tessellation of the nucleation centres (Fig. 13a), i.e., the grain boundary between grains I and $\mathrm{K}$ is the median line. Assuming globular grains, the smooth solid-liquid interfaces is first approximated by linear segment in each triangle linking a nucleation centre (open circle) and two vortices of the tessellation (open squares) (Fig. 13b). 


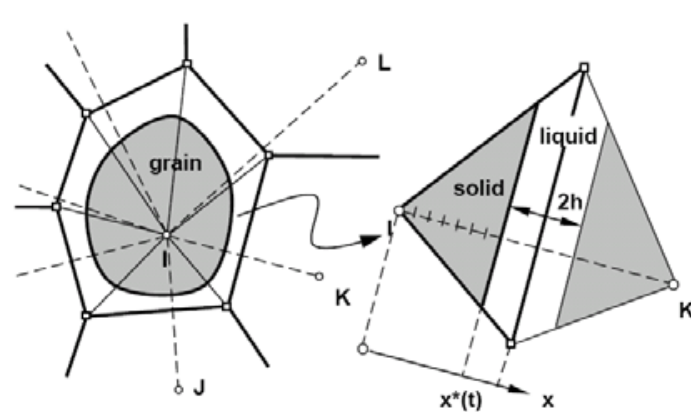

(a)

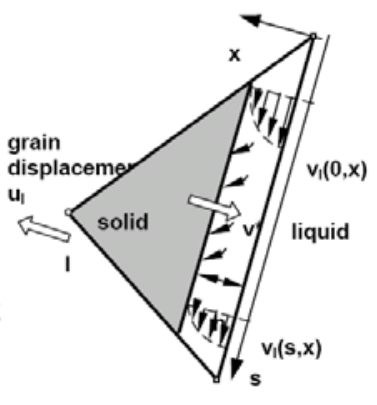

(c)

Figure 13: Schematics of the granular model used for the simulation of solidification and feeding in a network of equiaxed globular grains: Voronoï tessellation (a), microsegregation model (b), feeding KPL model (c).

Solidification is then calculated within each triangle using a microsegregation model [9]. Therefore, at any time, the remaining width of the liquid channel in between two grains is known providing the thermal field is known. When the two solid-liquid interfaces get within interaction distances, coalescence is accounted for, using Eq. (7) and a ReadSchockley grain boundary energy model. The next step is to calculate feeding within the network of liquid films. For that purpose, a Poiseuille flow was first assumed within the channels (Fig. 13c) [8,9]. This flow is not constant along a given channel as it has to feed solidification shrinkage and the relative movement of grains, i.e., the flow has some losses along each channel. Finally, at each vortex of the Voronoï tessellation, the sum of the (signed) incoming flows must be zero according to Kirchhoff's law (so-called Kirchoff Poiseuille with Losses, KPL, model).

The granular model has been applied to the directional solidification of an $\mathrm{Al}-1 \% \mathrm{Cu}$ alloy (Fig. 14). The thermal gradient was $60 \mathrm{~K} / \mathrm{cm}$ and the cooling rate $-1 \mathrm{~K} / \mathrm{s}$ (i.e., velocity of the isotherms equal to $170 \mu \mathrm{m} / \mathrm{s}$ ). The average grain density was set to $10^{8} \mathrm{~m}^{-2}$, i.e., average grain size of $100 \mu \mathrm{m}$, and the computation domain which spans across the whole mushy zone contains 14'000 grains. The central figure in Fig. 14 shows the grains with various grey levels, grains in solid contact (clusters) being represented with the same grey level while the liquid films are in black. The small figures on the left are magnifications of 4 typical regions of the mushy zone which are discussed hereafter. On the right of Fig. 14, the evolution of the volume fraction of solid as calculated in horizontal sections of the grain structure is represented together with the imposed temperature profile.

In region (a), typically for $0<\mathrm{g}_{\mathrm{s}}<0.89$, most the grains are isolated and surrounded by liquid films. For $0.89<\mathrm{g}_{\mathrm{s}}<0.97$ (region (b)), clusters 
of a few grains are formed but the liquid films remain continuous and interconnected. In region (c) characterised by $0.97<\mathrm{g}_{\mathrm{s}}<0.99$, larger clusters are visible, with a few isolated liquid films remaining inside. Finally, in region (d) $\left(0.99<\mathrm{g}_{\mathrm{s}}<1\right)$, the solid network is continuous and liquid only remains as isolated regions. As can be seen, this granular model is able to predict the gradual transition from a continuous intergranular film network to a continuous fully coherent solid. It should be emphasised that cluster formation is directly induced by the stochastic nature of the nucleation centre locations, a feature that has not been considered in past simulation works related to hot tearing. Further analysis of the transition regions is given in [11].

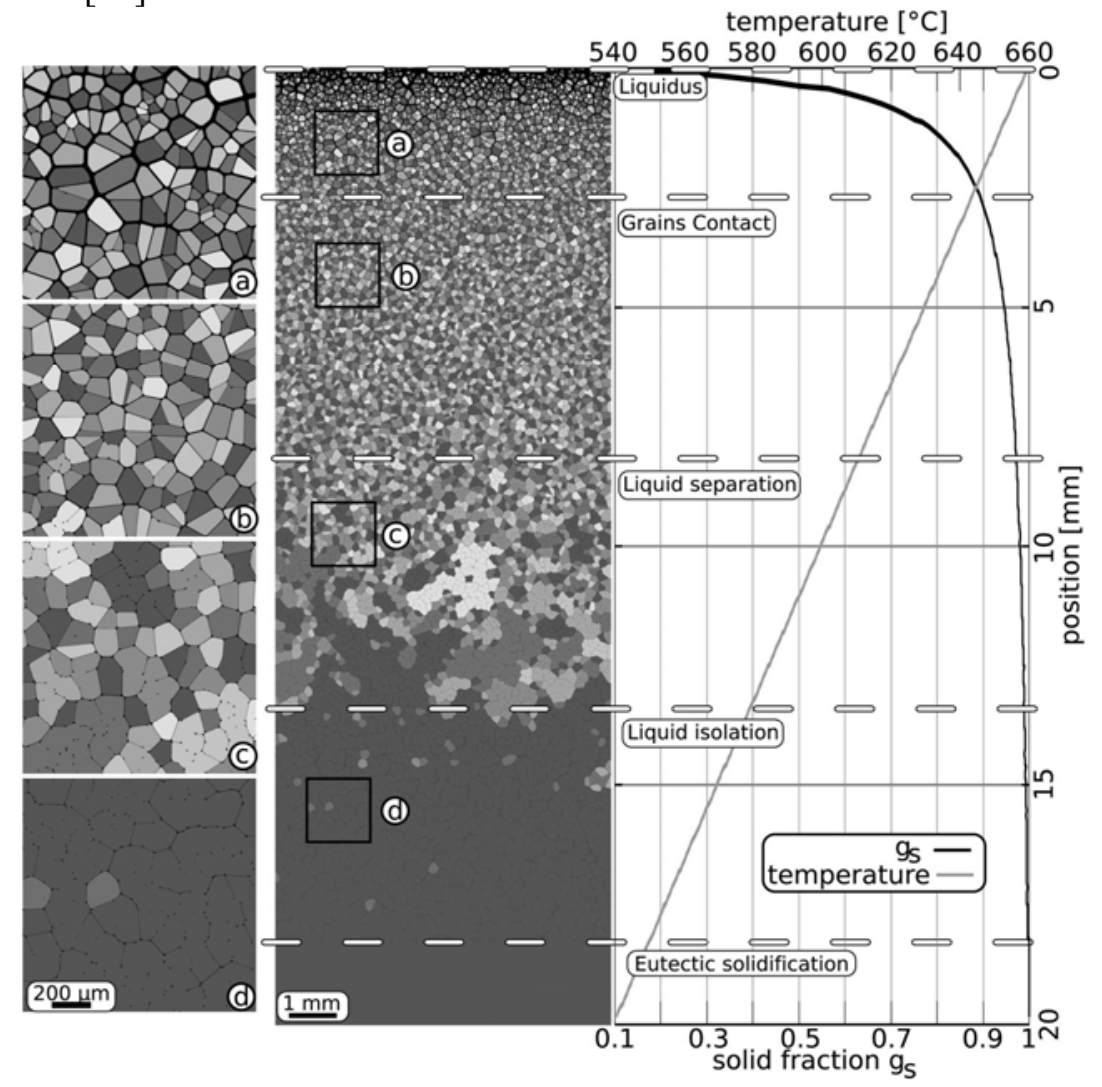

Figure 14: Calculated mushy zone for an $\mathrm{Al}-1 \mathrm{wt} \% \mathrm{Cu}$ alloy cooled down at -1 $\mathrm{K} / \mathrm{s}$ in a gradient of $6000 \mathrm{~K} / \mathrm{m}$. Grains in solid contact are shaded with the same grey level [11]. 


\section{Conclusion}

Hot tearing is a complex defect that involves many phenomena, in particular thermal and solidification aspects, stress-strain in an increasingly coherent solid, feeding in a gradually disappearing liquid film network. The RDG criterion provided the first two-phase approach, which was further improved using a more rigorous formalism and the complex rheology of porous media. Nevertheless, these approaches are still based on averages and do not consider any localization of strains and feeding at grain boundaries. Granular models, while still limited to small portions of a solidification process, have certainly the potential to answer some of these questions, once mechanical aspects will be fully built in and the model will be extended to 3 dimensions. The numerical simplicity of such approaches makes it feasible from a CPU time point of view.

\section{Acknowledgements}

The European Commission and the whole consortium of the Wel-Air project, Specific Targeted Research Project from the $6^{\text {th }}$ framework, are acknowledged for financial support. Isabelle Bordesoules from Alcan Péchiney CRV is greatly thanked for providing the Prophase computations.

\section{References}

[1] M. Rappaz, J.-M. Drezet and M. Gremaud: Met. Mat. Trans. 30A (1999), 449.

[2] J. Campbell: Castings (Elsevier, 2003).

[3] D.G. Eskin, Suyitno and L. Katgerman: Progr. Materials Science 49 (2004) 629.

[4] M. M'Hamdi, A. Mo, and C.L. Martin: Met. Mat. Trans. 33A (2002) 2081.

[5] M. M'Hamdi, H.G. Fjaer, A. Mo, D. Mortensen and S. Benum in TMS 2004.

[6] V. Mathier, J.-M. Drezet and M. Rappaz in Modeling of Casting, Welding and Advanced Solidification Processes, Eds. Ch. Gandin et al (TMS Publ., Warrendale, USA, 2006), to appear.

[7] V. Mathier, M. Rappaz and A. Jacot: Mod. Simul. Mater. Sc. Engng 12 (2004) 479.

[8] S. Vernède and M. Rappaz in Phil. Mag. (2006) to appear.

[9] S. Vernède and M. Rappaz: A Granular Model of Mushy Zones: Formation of a Coherent Solid and Localization of Feeding, to be published. 
[10] J.-M. Drezet and M. Rappaz in the proceedings of the First EsaForm Conference on Material Forming, Ecole des Mines de Paris, CEMEF, Sophia Antipolis, France, March 1998, pp. 49-52.

[11] M. Rappaz, J.-M. Drezet, V. Mathier and S. Vernède in Materials Science Forum vols. 519-521 (July 2006) pp. 1665-1674, Trans Tech Publications, Switzerland.

[12] E. Niyama, T. Uchida, M. Morikawa and S. Saito: AFS Int. Cast Metals J. (Sept.1982) 52.

[13] I. I. Novikov and O. E. Grushko in Materials Science and Technology, Sept. 1995, vol. 11, p. 926.

[14] B. Magnin et al. in Materials Science Forum vols. 217-22 (1996) pp. 1209 1214, Trans Tech Publications, Switzerland.

[15] W. Rindler, E. Kozeschnik and B. Buchmayr: Computer simulation of the Brittle Temperature Range (BTR) for hot cracking in steels, in Steel Res, 2000, vol. 71 (11),pp. 460-465.

[16] J.-M. Drezet, M. Gremaud, R. Graf and M. Gaümann, A new hot tearing criterion for steel, proceedings of the 4th European Continuous Casting Conference, IOM communications, Birmingham, UK, October 2002, pp. 755-763.

[17] W. Rindler, E. Kozeschnik, N. Enzinger and B. Buchmayr: A modified hot tearing criterion for steels, in Math. Modelling of Welding Phenomena 6, 2002, pp. 819-835.

[18] EU WelAIR project no.

[19] C. Sigli, R. Dif R., B. Commet and T. Warner in Materials Science Forum Vol. 426-432, Issue 1, 2003, Pages 351-356

[20] J.-M. Drezet et al. in Mathematical Modelling of Weld Phenomena 8, Ed. H. Cerjak.

[21] Calcosoft3D user manual, distributed by Calcom ESI, http://www.calcom.ch

[22] J.-M. Drezet and Michel Rappaz: "Prediction of hot tears in DC cast billets" in Light Metals, Ed. J.-L. Anjier, TMS, 2001, New Orleans, pp. 887-893.

[23] O. Ludwig, C.-L. Martin, J.-M. Drezet and M. Suéry in Met. Mat. Trans, vol 36A, June 2005, p. 1525-1535.

[24] Abaqus user manual, http://www.abaqus.com

[25] C. Monroe and C. Beckerman: Development of a hot tear indicator for steel castings, in Mat. Sc. and Eng. A, vol. 413-414 (2005), pp. 30-36.

[26] M. Rappaz, A. Jacot and W. J. Boettinger: Last-stage solidification of alloys: theoretical model of dendrite-arm and grain coalescence, in Met. and Mat. Trans. Vol. 34A, March 2003, pp. 467-479.

[27] N. Wang, S. Mokadem, M. Rappaz and W. Kurz: Acta Mater. 52 (2004) 3173. 